\title{
INCIDÊNCIA DE LESÕES E DESVIOS POSTURAIS EM ATLETAS DE TAEKWONDO'
}

\author{
MS. ALINE CAVALHEIRO TAMBORINDEGUY \\ Mestre em Ciências do Movimento Humano pela Escola de Educação Física da \\ Universidade Federal do Rio Grande do Sul (ESEF/UFRGS) \\ Doutoranda em Educação Física pelo Centro de Desportos da Universidade Federal \\ de Santa Catarina (CDS/UFSC) (Florianópolis - Santa Catarina - Brasil) \\ E-mail: aline.tambor@gmail.com \\ MS. ADRIANA SEÁRA TIRLONI \\ Mestre em Educação Física pelo Centro de Desportos da Universidade \\ Federal de Santa Catarina (CDS/UFSC) \\ Doutoranda em Engenharia de Produção pelo Centro Tecnológico \\ da Universidade Federal de Santa Catarina (CTC/UFSC) \\ (Florianópolis - Santa Catarina - Brasil) \\ E-mail: adri@tirloni.com.br
}

MS. DIOGO CUNHA DOS REIS

Mestre em Educação Física pelo Centro de Desportos da Universidade

Federal de Santa Catarina (CDS/UFSC)

Doutorando em Educação Física pelo Centro de Desportos

da Universidade Federal de Santa Catarina

(CDS/UFSC) (Florianópolis - Santa Catarina - Brasil)

E-mail: diogo.biomecanica@gmail.com

\section{Dra. CÍNTIA DE LA ROCHA FREITAS}

Doutora em Ciências do Movimento Humano pela Escola de Educação Física

da Universidade Federal do Rio Grande do Sul (ESEF/UFRGS)

Professora adjunta do Centro de Desportos da Universidade Federal de Santa Catarina (CDS/UFSC)

(Florianópolis - Santa Catarina - Brasil)

E-mail: cintia@cds.ufsc.br

\section{DR. ANTÔNIO RENATO PEREIRA MORO}

Doutor em Ciências do Movimento Humano pelo Centro de Educação Física e

Desportos da Universidade Federal de Santa Maria (CDS/UFSM)

Professor associado do Centro de Desportos da Universidade

Federal de Santa Catarina (CDS/UFSC)

(Florianópolis - Santa Catarina - Brasil)

E-mail:moro@cds.ufsc.br

I. Agradecimentos ao CNPq e à CAPES-REUNI pelas bolsas de doutorado. 


\author{
Dra. SARAY GIOVANA DOS SANTOS \\ Doutora em Engenharia de Produção pelo Centro Tecnológico da \\ Universidade Federal de Santa Catarina (CTC/UFSC) \\ Professora Associada do Centro de Desportos da \\ Universidade Federal de Santa Catarina (CDS/UFSC) \\ (Florianópolis - Santa Catarina - Brasil) \\ E-mail: saray@cds.ufsc.br
}

\begin{abstract}
RESUMO
Com o objetivo de analisar a incidência de lesões e desvios posturais no taekwondo foram avaliados 10 atletas do sexo masculino da seleção catarinense. O histórico de lesões relacionadas à prática desta modalidade foi obtido por aplicação de questionário. A postura foi analisada por meio do protocolo do software SAPO. Os resultados indicaram lesões mais frequentes nos membros inferiores (7/I0), principalmente no pé, por fratura e entorse. Houve diferença significativa entre os hemicorpos no ângulo $Q(p=0,037)$, ângulo perna/retropé $(p=0,00$ I), alinhamento horizontal da pelve $(p=0,016)$ e comprimento entre membros $(p=0,02)$. Sugere-se a implementação de treinamento preventivo e exercícios compensatórios para prevenir lesões e desvios posturais em atletas de taekwondo.
\end{abstract}

PALAVRAS-CHAVE: Artes marciais; lesões; postura; atletas.

\title{
INTRODUÇÃO
}

○ taekwondo, originado na Korea há mais de 1000 anos, é muito mais um esporte do que uma técnica de defesa pessoal (BUSCHBACHER; SHAY, 1999), o qual foi reconhecido como esporte oficial nas Olimpíadas de Sydney em 2000 (KAZEMI; SHEARER; CHOUNG, 2005). Esta modalidade esportiva é considerada global e simétrica tendo em vista a utilização de membros superiores e inferiores e do tronco em movimentos de ataque e defesa sempre associados a gestos de chute altamente dinâmicos em situações de instabilidade (LYSTAD; POLLARD; GRAHAM, 2009). Por ser o taekwondo um esporte cada vez mais competitivo, as valências físicas força, velocidade, equilíbrio, resistência e coordenação são atributos importantes para o atleta desta modalidade (MELHIM, 200 I).

A prática de métodos sistematizados de treinamento gera adaptações orgânicas necessárias para a otimização do desempenho (SILVA, 2008). De forma geral, nos esportes a realização de movimentos repetitivos com a sobrecarga de treinamento leva a um processo de adaptação orgânica que pode resultar em efeitos deletérios para a postura, com alto potencial de desequilíbrio muscular (RIBEIRO et al., 2003). Além disso, a especificidade do esporte e a possibilidade de erros na técnica de execução dos movimentos podem aumentar a prevalência de lesões (BASTOS et al., 2009; 
GUIMARÃES; SACCO; JOÃO, 2007; NETO JÚNIOR; PASTRE; MONTEIRO, 2004). Em razão das características do gesto específico do esporte, os atletas de taekwondo são especialmente expostos a riscos frequentes de lesões (ESTEVES; REIS; SANTOS, 2006). Apesar da maioria das lesões encontradas por Lystad, Pollard e Graham (2009) em lutadores desta modalidade esportiva ocorrer devido ao contato, grande parte delas é classificada como sendo de severidade mínima a moderada.

Estudos indicam que o mecanismo mais comum de lesão relatado no taekwondo é o receber um chute (defesa) e pelo movimento de dar um chute (ataque) (KAZEMI; PIETER, 2004; KAZEMI; SHEARER; CHOUNG, 2005; KAZEMI et al., 2009; LYSTAD; POLLARD; GRAHAM, 2009). No entanto, existem discussões sobre os fatores que intervêm na ocorrência de lesões. Kazemi et al. (2009) verificaram que o nível de experiência do competidor interferiu diretamente na ocorrência de lesão, enquanto que o estudo desenvolvido por Lystad, Pollard e Graham (2009) indicou que a idade, o sexo e o nível de prática esportiva do atleta não interferiram no risco de lesão.

Em atletas de taekwondo têm sido investigadas: a capacidade anaeróbica e aeróbica (LIN et al., 2006; MELHIM, 200I); as adaptações fisiológicas e esforço percebido durante competição (BOUHLEL et al., 2006; BRIDGE et al., 2007) e treinamento (BRIDGE et al., 2007); os aspectos biomecânicos do gesto esportivo (ESTEVES, REIS, SANTOS, 2006; FALCO et al., 2009); a utilização de equipamentos de proteção (BURKE et al., 2003); a incidência de lesões (LYSTAD; POLLARD; GRAHAM, 2009) e as características físicas e os hábitos de treinamento que predispõem os atletas a lesões (KAZEMI et al., 2009). Contudo, carecem estudos relacionados às alterações posturais dos atletas desta modalidade.

A análise da postura estática é foco de investigação em diferentes modalidades esportivas como vôlei de quadra e de praia, tênis de mesa, natação (OLIVEIRA; DEPRÁ, 2005), futsal (RIBEIRO et al., 2003), ginástica artística (GUIMARÃES; SACCO; JOÃO, 2007), ginástica rítmica (SILVA, 2008), corredores e saltadores (BASTOS et al., 2009) e atletas de provas de potência muscular (NETO JÚNIOR; PASTRE; MONTEIRO, 2004). Nestes casos os autores buscam a compreensão da relação do treinamento esportivo, da iniciação precoce ao esporte e da prevalência de lesões com alterações posturais. A maioria desses estudos (BASTOS et al., 2009; GUIMARÃES; SACCO; JOÃO, 2007; RIBEIRO et al., 2003; SILVA, 2008) discute que as diferentes modalidades esportivas desenvolvem como forma adaptativa ao treinamento intenso e por longo período, padrões posturais característicos correspondentes ao gesto motor proeminente. 
A partir da panorâmica atual das exigências do taekwondo competitivo e da constatação de uma lacuna na literatura, surge a problemática do presente estudo: Existe um padrão postural e de tipos de lesão característicos do atleta de taekwondo? Diante do exposto, este estudo teve como objetivo analisar a incidência de lesões e desvios posturais em atletas de taekwondo. Mais especificamente objetivou-se verificar a ocorrência e os tipos de lesões, as regiões corporais acometidas, os mecanismos causadores e a frequência de lesão para cada região corporal decorrentes da prática do taekwondo; e identificar e comparar as características da postura estática nos planos frontal e sagital de atletas da seleção catarinense de taekwondo.

\section{MATERIAL E MÉTODOS}

Participaram deste estudo 10 atletas do sexo masculino, selecionados de forma não probabilística intencional por voluntariado, que faziam parte da seleção catarinense de taekwondo com 25,0 \pm 3,0 anos de idade, 67,3 $\pm 10,6 \mathrm{~kg}$ de massa corporal e 176,6 \pm 10,3 cm de estatura. Praticavam taekwondo há 6,6 \pm 4,2 anos, com frequência semanal de treinamento de 4,4 \pm I,3 sessões por semana e a duração das sessões entre 60 e 120 minutos. Realizavam treinamento aeróbio (4/I0) e de flexibilidade (4/I0), sendo a musculação realizada por apenas dois atletas. A maioria dos atletas $(7 / / 0)$ não era submetida a avaliações neuromusculares periodicamente e nenhum deles realizava trabalho compensatório e preventivo.

Foi verificado que a maioria $(7 / 10)$ dos atletas apresentou dominância lateral superior e inferior direita. Dos demais atletas, dois relataram dominância lateral superior esquerda, um relatou ser ambidestro para membros superiores, sendo que estes três tinham dominância lateral inferior esquerda.

O presente estudo foi aprovado pelo Comitê de Ética local, sob número de processo 609/10. Previamente à realização das coletas de dados, os atletas foram esclarecidos sobre os objetivos do estudo e então assinaram um termo de consentimento livre e esclarecido.

Para a caracterização dos atletas, a verificação da frequência, a duração, o tipo de treinamento, a realização de avaliações físicas, a dominância lateral e o tempo de prática do esporte, assim como a presença de lesões decorrentes da prática esportiva, seus mecanismos causais e a frequência de lesão para cada região corporal, foi aplicado um questionário estruturado adaptado do formulado por Santos, Piucco e Reis (2007).

Foram utilizadas duas câmeras fotográficas digitais (Sony ${ }^{\circledR}$ DSC-P52 e Canon ${ }^{\circledR}$ A590IS) para aquisição das imagens dos atletas para a avaliação postural. As câmeras foram posicionadas perpendicularmente ao avaliado, a 3,0 m de distância e I,06 m 
de altura. Uma das câmeras foi utilizada para a aquisição das imagens no plano sagital (vistas direita e esquerda) e a outra no plano frontal (vistas posterior e anterior). Para a calibração do software foi colocado um fio de prumo perpendicularmente ao chão, sendo utilizado 1,0 m de seu comprimento.

A avaliação postural foi realizada por meio do software de avaliação postural - SAPO $0.68^{20}$. Previamente à aquisição das fotografias foram demarcados com semiesferas de isopor ( $\varnothing=15 \mathrm{~mm}$ ), os pontos anatômicos (trago, acrômio, espinha ilíaca ântero-superior, trocânter maior do fêmur, linha articular do joelho, ponto medial da patela, tuberosidade da tíbia, maléolo lateral e medial, ponto entre a cabeça do $2^{\circ}$ e $3^{\circ}$ metatarso, processos espinhosos C7 e T3, ângulo inferior da escápula, espinha ilíaca póstero-superior, ponto sobre a linha média da perna, ponto sobre o tendão do calcâneo na altura média dos maléolos e calcâneo) preconizados pelo protocolo do SAPO para ambos os hemicorpos, por meio de palpação.

A análise estatística foi realizada com o software SPSS ${ }^{\circledR}$ for Windows versão I 5 (SPSS Inc., Chicago IL, EUA). Os dados foram apresentados por meio de estatística descritiva (média e desvio-padrão). Depois de constatada a normalidade dos dados por meio do teste de Shapiro-Wilk, utilizou-se o teste "t" de Student pareado $(p \leq 0,05)$ para comparar as variáveis bilaterais da postura estática (ângulo $Q$, ângulo perna/retropé, alinhamento horizontal da pelve, comprimento de membros inferiores e perímetro de coxa).

\section{RESULTADOS}

A maioria (6/10) dos atletas sentia algum tipo de desconforto corporal após a competição ou sessão de treino intenso no quadril (2), na coxa (2), no joelho (I), na perna (2) e/ou no tornozelo ( 1 ), sendo que quatro atletas citaram que os desconfortos foram causados pelo esforço físico, exercícios de alongamento, pancadas ou treinos frequentes, e dois não souberam definir as causas.

Constatou-se que a maioria (8/10) dos atletas já teve algum tipo de lesão aguda advinda da prática do taekwondo, incidindo principalmente nos membros inferiores, sendo a região do pé a mais acometida $(7 / 10)$ por entorse e fratura (Tabela I). Diferentemente da lesão aguda, a lesão crônica foi citada por apenas um atleta e referia-se a região do quadril. Também foi relatado por sete dos atletas que apresentaram lesões, que o retorno aos treinos era sintomático, e três atletas necessitaram de afastamento dos treinamentos para tratamento da lesão.

$\mathrm{Na}$ Tabela I estão descritos os tipos de lesões agudas citadas pelos atletas, as regiões corporais e o hemicorpo onde ocorreram as lesões, os mecanismos de lesão e a frequência de vezes em que as lesões ocorreram. 
Tabela I - Descrição das lesões, região corporal, hemicorpo acometido, mecanismo e frequência de lesão para cada região corporal dos atletas catarinenses de taekwondo

\begin{tabular}{|c|c|c|c|c|c|c|}
\hline Atletas & Tipos de lesão & $\begin{array}{l}\text { Região } \\
\text { corporal }\end{array}$ & Direito & Esquerdo & $\begin{array}{l}\text { Mecanismo } \\
\text { de lesão }\end{array}$ & $\begin{array}{l}\text { Fre- } \\
\text { quência }\end{array}$ \\
\hline I & $\begin{array}{l}\text { Distensão muscular } \\
\text { Ruptura ligamentar } \\
\text { Fratura }\end{array}$ & $\begin{array}{l}\text { Coxa } \\
\text { Joelho } \\
\text { Pé }\end{array}$ & $\begin{array}{l}x \\
x\end{array}$ & $\begin{array}{l}x \\
x\end{array}$ & $\begin{array}{l}\text { Ataque } \\
\text { Ataque } \\
\text { Ataque }\end{array}$ & $\begin{array}{l}1 \\
2 \\
1\end{array}$ \\
\hline 2 & $\begin{array}{l}\text { Entorse } \\
\text { Fratura }\end{array}$ & $\begin{array}{l}\text { Tornozelo } \\
\text { Pé }\end{array}$ & $x$ & $x$ & Ataque & i \\
\hline 3 & $\begin{array}{l}\text { Dor aguda inespecífica } \\
\text { Dor aguda inespecífica } \\
\text { Dor aguda inespecífica } \\
\text { Dor aguda inespecífica } \\
\text { Dor aguda inespecífica } \\
\text { Fratura } \\
\text { Dor aguda inespecífica } \\
\text { Distensão muscular } \\
\text { Contratura muscular } \\
\text { Dor aguda inespecífica } \\
\text { Dor aguda inespecífica } \\
\text { Fratura }\end{array}$ & $\begin{array}{l}\text { Braço } \\
\text { Antebraço } \\
\text { Cotovelo } \\
\text { Punho } \\
\text { Mão } \\
\text { Tórax } \\
\text { Região lom- } \\
\text { bar } \\
\text { Virilha } \\
\text { Coxa } \\
\text { Joelho } \\
\text { Tornozelo } \\
\text { Pé }\end{array}$ & $\begin{array}{l}x \\
x \\
x \\
x \\
x\end{array}$ & $\begin{array}{l}x \\
x \\
x \\
x \\
x \\
x \\
x\end{array}$ & $\begin{array}{l}\text { Defesa } \\
\text { Defesa } \\
\text { Defesa } \\
\text { Defesa } \\
\text { Defesa } \\
\text { Defesa } \\
\text { Ataque } \\
\text { Ataque/de- } \\
\text { fesa } \\
\text { Ataque } \\
\text { Ataque } \\
\text { Ataque } \\
\text { Contra- } \\
\text { ataque }\end{array}$ & $\begin{array}{l}4 \\
3 \\
2 \\
3 \\
4 \\
1 \\
2 \\
3 \\
2 \\
2 \\
2 \\
4\end{array}$ \\
\hline 4 & $\begin{array}{l}\text { Distensão muscular } \\
\text { Distensão muscular } \\
\text { Entorse }\end{array}$ & $\begin{array}{l}\text { Cervical } \\
\text { Coxa } \\
\text { Pé }\end{array}$ & $\begin{array}{l}x \\
x\end{array}$ & $\begin{array}{l}x \\
x\end{array}$ & $\begin{array}{l}\text { Ataque } \\
\text { Ataque } \\
\text { Ataque }\end{array}$ & $\begin{array}{l}1 \\
2 \\
2\end{array}$ \\
\hline $\begin{array}{l}5 \\
6\end{array}$ & $\begin{array}{l}\text { Sem lesão } \\
\text { Sem lesão }\end{array}$ & - & - & - & - & - \\
\hline 7 & $\begin{array}{l}\text { Distensão muscular } \\
\text { Entorse }\end{array}$ & $\begin{array}{l}\text { Cotovelos } \\
\text { Pé }\end{array}$ & $x$ & $\begin{array}{l}x \\
x\end{array}$ & $\begin{array}{l}\text { Defesa } \\
\text { Ataque }\end{array}$ & $\begin{array}{l}4 \\
1\end{array}$ \\
\hline 8 & Entorse & Pé & & $x$ & Ataque & I \\
\hline 9 & Não especificado & $\begin{array}{l}\text { Antebraço } \\
\text { Cotovelo } \\
\text { Punho } \\
\text { Mão } \\
\text { Coxa } \\
\text { Joelho } \\
\text { Perna } \\
\text { Pé }\end{array}$ & $\begin{array}{l}x \\
x \\
x\end{array}$ & $\begin{array}{l}x \\
x \\
x \\
x \\
x \\
x \\
x \\
x \\
x\end{array}$ & $\begin{array}{l}\text { Defesa } \\
\text { Defesa } \\
\text { Defesa } \\
\text { Defesa } \\
\text { Ataque/de- } \\
\text { fesa } \\
\text { Ataque } \\
\text { Ataque } \\
\text { Ataque }\end{array}$ & $\begin{array}{c}10 \\
10 \\
3 \\
3 \\
9 \\
3 \\
3 \\
10\end{array}$ \\
\hline 10 & Dor aguda inespecífica & Joelho & & $x$ & $\begin{array}{l}\text { Impacto do } \\
\text { salto }\end{array}$ & । \\
\hline
\end{tabular}

Na Tabela 2 estão apresentados os resultados da avaliação postural dos atletas de taekwondo, na qual foram abordados os alinhamentos corporais, as assimetrias, os ângulos perna/retropé e o posicionamento do centro de gravidade. 
Tabela 2 - Resultados da avaliação postural dos atletas catarinenses de taekwondo por meio do software SAPO

\begin{tabular}{|c|c|c|c|c|c|c|c|c|c|c|c|}
\hline \multirow[b]{2}{*}{ Vista anterior } & \multicolumn{11}{|c|}{ Atletas } \\
\hline & 1 & 2 & 3 & 4 & 5 & 6 & 7 & 8 & 9 & 10 & $\overline{\mathbf{X}} \pm s$ \\
\hline $\mathrm{AHA}\left({ }^{\circ}\right)$ & 1,6 & 2 & 1,9 & -1 & $-0,7$ & 4,2 & $-1,2$ & 2,9 & 1,3 & 1,9 & $1,3 \pm 1,8$ \\
\hline $\operatorname{AHEIA~}\left({ }^{\circ}\right)$ & 3,2 & 0,7 & I & 2,3 & ।,7 & $-0,3$ & 1,9 & $-0,3$ & 3,9 & 0 & $1,4 \pm 1,5$ \\
\hline $\begin{array}{l}\text { DIFMMII } \\
(\mathrm{cm})\end{array}$ & $-1,8$ & $-0,9$ & $-0,2$ & $-0,4$ & $-0,8$ & $-0,3$ & -1 & $-0,2$ & $-1,8$ & $-0,4$ & $-0,8 \pm 0,6$ \\
\hline $\operatorname{AHTT}\left(^{\circ}\right)$ & 0,3 & $-1,7$ & 0,7 & 2,4 & -1 & 0,3 & 1,4 & $-1,7$ & 2 & -1 & $0,2 \pm 1,5$ \\
\hline $\operatorname{AQD}\left({ }^{\circ}\right)$ & 16 & 10,8 & 11,3 & 17 & 29,3 & 24,2 & 25,3 & 16,3 & 16,1 & 12,3 & $17,9 \pm 6,3^{\mathrm{A}}$ \\
\hline $\mathrm{AQE}\left({ }^{\circ}\right)$ & 12,7 & 15,5 & 5,9 & 11,4 & 15,5 & 24,6 & 8,9 & 3,8 & 12,3 & 14,1 & $12,5 \pm 5,8^{B}$ \\
\hline \multicolumn{12}{|l|}{ Vista posterior } \\
\hline IAT3 (\%) & 5,3 & $-7,8$ & $-2,7$ & 17,5 & 5,1 & 8,6 & 8,3 & $-|5|$, & 9,8 & 1,3 & $3,0 \pm 9,5$ \\
\hline $\operatorname{APD}\left({ }^{\circ}\right)$ & $-3,1$ & 9,4 & $-0,2$ & 3,5 & 4,0 & 0,8 & 2,5 & 0,6 & 0,6 & $-5,0$ & $1,3 \pm 4,0^{A}$ \\
\hline $\operatorname{APE}\left({ }^{\circ}\right)$ & 2,1 & 11,5 & 5,8 & 5,3 & 4,0 & 7,8 & 4,2 & 6,7 & 4,0 & 3,8 & $5,5 \pm 2,7^{в}$ \\
\hline \multicolumn{12}{|l|}{$\begin{array}{l}\text { Vistas } \\
\text { Laterais }\end{array}$} \\
\hline $\operatorname{AHPD}\left({ }^{\circ}\right)$ & $-12,3$ & $-9,7$ & $-3,8$ & $-8,2$ & $-8,4$ & $-16,1$ & $-2,8$ & $-11,1$ & $-18,4$ & -10 & $-10,1 \pm 4,8^{A}$ \\
\hline $\operatorname{AHPE}\left(\left(^{\circ}\right)\right.$ & -5 & $-5,8$ & $-5,6$ & $-7,8$ & $-7,8$ & $-10,4$ & $-2,5$ & $-9,9$ & $-12,5$ & $-4,3$ & $-7,2 \pm 3,\left.\right|^{B}$ \\
\hline $\begin{array}{l}\text { Assimetria } \\
\text { frontal (\%) }\end{array}$ & $-1,1$ & 5,8 & $1 \mid, 4$ & 7,2 & 13,5 & 9,7 & 2,9 & 0,5 & 9,7 & $-9,6$ & $5,0 \pm 7,0$ \\
\hline $\begin{array}{l}\text { Assimetria } \\
\text { sagital (\%) }\end{array}$ & 39,6 & 30,6 & 40,5 & 35,7 & 23,4 & 31,4 & 15,6 & 24,5 & 34,7 & 18,5 & $29,5 \pm 8,6$ \\
\hline $\begin{array}{l}\text { Posição CG } \\
\text { frontal }(\mathrm{cm})\end{array}$ & $-0,1$ & 0,8 & 1,8 & 0,8 & 2 & I,4 & 0,5 & 0,1 & 1,2 & $-1,3$ & $0,7 \pm 1,0$ \\
\hline $\begin{array}{l}\text { Posiçãa CG } \\
\text { lateral (cm) }\end{array}$ & 6,4 & 3,9 & 6,8 & 5,3 & 3,5 & 4,2 & 2,1 & 3,5 & 5 & 2,8 & $4,4 \pm 1,5$ \\
\hline
\end{tabular}

Legenda: AHA - Alinhamento horizontal dos acrômios; AHEIAS - Alinhamento horizontal das espinhas ilíacas ânterosuperiores; DIFMMII - Diferença entre os membros inferiores direito e esquerdo (D - E); AVC (acrômio): alinhamento vertical da cabeça em relação ao acrômio; AHTT - Alinhamento horizontal das tuberosidades das tíbias; AQD - Ângulo Q direito; AQE - Ângulo Q esquerdo; IAT3 - Índice de assimetria em relação a T3; APD - Ângulo perna/retropé direito; APE - Ângulo perna/retropé esquerdo; AHPD - Alinhamento horizontal da pelve direita; AHPE - Alinhamento horizontal da pelve esquerda; CG - Centro de gravidade; Valores positivos - desvio no sentido anti-horário (lado esquerdo do corpo mais elevado que o direito), projeção do corpo anteriorizada ou para direita; Letras diferentes identificam diferenças significativas $(p \leq 0,05)$.

Conforme a Tabela 2, foram constatadas diferenças significativas entre os hemicorpos nas variáveis: ângulo $Q(p=0,037)$, ângulo perna/retropé $(p=0,00$ I $)$ e alinhamento horizontal da pelve $(p=0,016)$. A maioria dos atletas $(8 / 10)$ apresentou assimetria no plano frontal para a direita e, no plano sagital, todos estavam anteriorizados.

Também foi verificada diferença significativa $(p=0,02)$ entre os comprimentos de membros inferiores direito $(84,6 \pm 5,1 \mathrm{~cm})$ e esquerdo $(85,4 \pm 5,1$ $\mathrm{cm}$ ) dos atletas, sendo que todos apresentaram o membro esquerdo maior que o direito (Tabela 2).

Ao mensurar o perímetro de coxa, constatou-se que não existiu diferença significativa $(p=0,121)$ entre os perímetros de coxa direita $(51,7 \pm 4,5 \mathrm{~cm})$ e esquerda $(51,3 \pm 4,0 \mathrm{~cm})$ dos atletas. 


\section{DISCUSSÃO}

Ao verificar a ocorrência de 904 lesões em 58 competições no decorrer de 9 anos em 664 lutadores de taekwondo do Canadá, Kazemi et al. (2009) observaram que os atletas mais experientes apresentaram um maior número de lesões recidivantes. Por outro lado, observaram que as lesões mais graves e causadas por chute defensivo ocorreram em maior parte nos atletas menos experientes (54\%), de faixas coloridas, do que nos mais experientes (40\%), de faixa preta. Segundo os autores, esses resultados relacionaram-se com maiores níveis de agressividade impostos durante golpes de atletas menos experientes, como forma de compensar deficiências técnicas. Desta forma, o aumento da agressividade durante os golpes levaria a um aumento da sobrecarga física imposta ao corpo do atleta que ataca e, consequentemente, ao que defende. Kazemi et al. (2009) sugerem ainda, que ao classificar um atleta como experiente seja considerada a quantidade de tempo gasto pelo mesmo com o treinamento. No presente estudo, o treinamento dos atletas se assemelhara aos do estudo de Kazemi, Shearer e Choug (2005) que teve 20 dos 28 atletas praticando entre uma e duas horas por sessão.

O treinamento intenso e a participação em competições de taekwondo podem levar à fadiga e causar desconforto nos membros inferiores (BRIDGE; JONES; DRUST, 2009), como relatado no presente estudo. Segundo os autores, estes segmentos são intensamente solicitados durante uma competição tendo em vista que o objetivo é nocautear o oponente com o maior número de pontos por meio da execução de chutes. Desta forma, segundo Lystad, Pollard e Graham (2009), as sobrecargas mecânicas que decorrem da luta relacionam-se à alta exigência de esforços musculares e ao impacto do contato ao atacar e ao defender, podendo inclusive provocar lesões (ESTEVES; REIS; SANTOS, 2006).

No presente estudo, a maior ocorrência de lesões agudas também foi em membros inferiores, principalmente no pé, com fraturas ou entorses. Estes resultados corroboram com os dados de Gartland et al. (200 I) envolvendo outra arte marcial que utiliza muito os membros inferiores, o Muay Thay. Os mesmos autores mencionam que em iniciantes e amadores a segunda lesão mais comum foi entorse e distensão muscular, enquanto que em atletas profissionais foi a fratura, destacando que a maioria das lesões ocorreu em membros inferiores.

Por outro lado, os resultados do estudo de Kazemi et al. (2009) indicam que as três regiões corporais mais comuns de lesão em lutadores de taekwondo foram cabeça, pé e coxa. O estudo de Kazemi e Pieter (2004), realizado com 3 I 8 atletas adultos canadenses do mesmo esporte, observou que o maior número de lesões, nos homens, ocorreu nos membros inferiores e o tipo mais comum de lesão foi a 
entorse, dados que corroboram com os resultados do presente estudo. Kazemi et al. (2005) também encontraram o maior número de lesões em membros inferiores e o segundo maior número de lesões em membros superiores. A região da cabeça, por sua vez, foi a menos afetada. Esses achados levam a crer que equipamentos de proteção de antebraço não estão sendo utilizados ou não estão sendo efetivos em sua função, tendo em vista a grande possibilidade das lesões de membro superior ser decorrente do contato durante o ato de defesa de chutes nesta região. A grande maioria das lesões sofridas pelos competidores foi causada tanto por chutes defensivos quanto ofensivos. Isto era esperado, haja vista que o taekwondo tem o foco no uso dos membros inferiores como instrumento de ataque (ESTEVES; REIS; SANTOS, 2006; KAZEMI et al., 2009).

Devido às características do esporte em questão, a maior parte das lesões é relacionada à contusão e entorse em membros inferiores (KAZEMI; SHEARER; CHOUNG, 2005; LYSTAD; POLLARD; GRAHAM, 2009). Beis et al. (2007) sugerem o aprimoramento das técnicas de bloqueio para reduzir a incidência de lesões. Além disso, salienta-se a importância da implementação de medidas de segurança (equipamentos) para a prática do taekwondo como forma de reduzir os riscos de lesão (BURKE et al., 2003) e o controle da utilização de alongamentos antes ou depois do aquecimento como forma de prevenção das lesões (KAZEMI; SHEARER; CHOUNG, 2005).

Entre os dez atletas avaliados no presente estudo, sete retornaram aos treinos após a lesão ainda sentindo dor. Estes resultados corroboram com estudos realizados em outras modalidades, tal como o de Pastre et al. (2004), os quais relataram que 49 dos 60 atletas de atletismo avaliados tiveram retorno sintomático ao esporte. Para Ejnisman et al. (200 I), o atleta, na maioria das vezes, consegue praticar a atividade com dor de fraca intensidade, não a suspendendo muitas vezes para não perder o lugar na equipe ou por não conseguir atendimento médico e fisioterapêutico adequado. Apesar de apenas um dos atletas avaliado no presente estudo referir lesão crônica, é importante alertar sobre a necessidade do tratamento adequado dos atletas lesionados, respeitando o período necessário para cicatrização tecidual e recuperação das lesões. As entorses de tornozelo, por exemplo, lesões prevalentes no estudo atual, são as lesões esportivas mais frequentes, e ainda podem levar à dor crônica, edema e instabilidade funcional devendo, portanto, ser prevenidas principalmente por exercícios de propriocepção (OSBORNE; RIZZO, 2003).

A ocorrência de lesões de atletas pode ser relacionada às características posturais. Conforme pode ser observado no estudo de Ribeiro et al. (2003), ao avaliarem 50 meninos praticantes de futsal com idades entre 9 e 16 anos, apenas I/27 atletas com lesão relacionada a prática do futebol de salão apresentou 
alinhamento normal da lordose lombar, enquanto que 5/23 meninos sem lesão apresentaram alinhamento normal da lordose lombar (diferença significativa entre os grupos, $p \leq 0,05$ ). Além disso, foi observado que a distribuição das lesões por segmento coincidia com a região dos desvios posturais. Segundo esses autores, a incidência de desvios posturais está relacionada ao aumento no risco de lesão, uma vez que o desalinhamento postural gera sobrecarga extra em músculos específicos e, consequentemente na articulação, solicitando o segmento de maneira biomecanicamente incorreta, criando estresse e estiramento de partes moles, diminuindo a eficiência muscular e ligamentar.

A busca por uma caracterização postural relacionada à especificidade do esporte tem sido alvo de estudos (GUIMARÃES; SACCO; JOÃO, 2007; JÚNIOR; PASTRE; MONTEIRO, 2004; OLIVEIRA; DEPRÁ, 2005; RIBEIRO et al., 2003; SILVA, 2008; WOJTYS et al., 2000). Ao comparar a postura de meninas de 8 a 12 anos de idade, 39 praticantes de ginástica olímpica e 48 não praticantes, foi observado que as jovens atletas apresentaram um melhor alinhamento da postura do membro inferior. Porém, as atletas apresentaram um aumento da inclinação pélvica anterior bem como uma tendência ao aumento da lordose lombar (GUIMARÃES; SACCO; JOÃO, 2007).

Júnior, Pastre e Monteiro (2004), ao descreverem o perfil postural de I5 atletas do sexo masculino, especializados em provas de potência, relataram a ocorrência de tornozelo em valgo em $67 \%$ dos casos, rotação interna do quadril à direita em 60\%, lado esquerdo mais elevado em 47\%. Tais alterações foram relacionadas com a corrida em curva que sobrecarrega a estrutura da pelve para a manutenção da velocidade com simultânea mudança de direção em função da força gravitacional. Apontaram ainda anteversão pélvica em 73\% das avaliações decorrendo de retração observada nos músculos flexores do quadril e extensores do joelho e contribuindo para a formação de hiperlordose lombar (observada em $73 \%$ dos casos), retração da cadeia posterior com cifose torácica (53\%) e cabeça em protrusão (73\%).

Segundo Bastos et al. (2009), entre as diversas modalidades do atletismo são adotados níveis distintos de postura. Atletas de velocidade e de resistência apresentaram varo de joelho e tornozelo valgo. Especialistas em saltos, por sua vez, mostraram predominantemente tronco equilibrado, anteversão pélvica e joelho varo. Arremessadores/lançadores apresentaram tronco em posição de equilíbrio, rotação esquerda de quadril, joelho normal e tornozelo valgo.

Oliveira e Deprá (2005) encontraram em 37 atletas de variadas modalidades, pelo menos um desvio postural moderado. Dentre estes, os atletas de voleibol de quadra apresentaram o maior percentual e a natação o menor percentual de desvios 
moderados. A alteração postural mais comum nos atletas foi escápulas abduzidas (desvio moderado) e hipercifose (desvio acentuado).

O taekwondo, apesar de ser um esporte bilateral, pode predispor a desequilíbrios posturais como resposta adaptativa à repetição dos movimentos específicos deste esporte. No presente estudo, foi observada diferença significativa no valor médio do ângulo $Q$ do joelho direito (17,9 \pm 6,3 graus) em relação ao esquerdo ( $12,5 \pm 5,8$ graus). Ao classificar os valores do ângulo $Q$, pode-se observar que o joelho direito estava acima do normal tanto para o critério de Belchior et al. (2006) ( $>13^{\circ}$ para homens) quanto para a referência utilizada pelo protocolo do software SAPO 0.68 ( $>15^{\circ}$ para ambos Os sexos) (FUNDAÇÃO DE AMPARO À PESQUISA DO ESTADO DE SÃO PAULO, 2003). O aumento deste ângulo gera um maior vetor de força em valgo e aumenta a tração lateral da patela, aumentando a pressão na sua faceta lateral (BELCHIOR et al., 2006). Para estes autores este desequilíbrio pode predispor a subluxação patelar, amolecimento da cartilagem e estresse do retináculo patelar, além de contribuir para a disfunção femoropatelar.

Geralmente durante as sessões de treinamento, os gestos técnicos são realizados bilateralmente. No entanto, durante a luta, o maior número de chutes pode ocorrer com o membro inferior dominante. Pode-se inferir que, a preferência pelo chute com o membro inferior direito em sete dos dez atletas avaliados, esteja relacionada ao aumento do ângulo $Q$, tendo em vista, as frequentes repetições da aplicação de forças em valgo impostas à extremidade inferior durante os chutes.

Na vista posterior (Tabela 2), a diferença significativa observada no ângulo da perna-retropé entre os hemicorpos indicam supinação do calcâneo no lado esquerdo. Este posicionamento em supinação do calcâneo do membro inferior de apoio na maioria dos atletas pode estar relacionado aos constantes movimentos de transferência de peso característicos do esporte. Outro fator importante a ser considerado, é que o calcâneo supinado pode predispor a entorse de tornozelo por inversão.

Quanto ao alinhamento horizontal da pelve na vista lateral, constatou-se que os valores foram negativos tanto na visualização pelo lado direito quanto pelo esquerdo. Entretanto, estes valores são interpretados como retroversão para o lado esquerdo e anteversão para o direito. Hipotetiza-se que tais diferenças sejam representativas de desequilíbrio pélvico, que pode ser observada pela variação dos valores de alinhamento horizontal das espinhas ilíacas ântero-superiores. Apesar de não ter sido analisada a rotação pélvica no presente estudo, os desequilíbrios pélvicos encontrados podem ser justificados pela colocação de Lystad, Pollard e Graham (2009), ao afirmarem que as transferências de peso corporal e rotações ocorrem com frequência durante a luta exigindo esforços de estabilização muscular sobre as articulações. 
Ao analisar o posicionamento frontal do centro de gravidade dos atletas, foi observada a transferência do apoio corporal de todos os atletas no membro inferior direito, possivelmente como forma de compensar o maior comprimento do membro oposto, na tentativa de amenizar os desequilíbrios. Essa suposição se confirma com o fato de oito entre dez avaliados apresentarem assimetria no plano frontal para a direita.

Durante a luta são frequentes as transferências de peso entre os membros inferiores (LYSTAD; POLLARD; GRAHAM, 2009) e a perna de apoio é a que permanece mais tempo sustentando o corpo do atleta durante o chute, gesto essencial da modalidade. Segundo Pereira (200 I), o treinamento esportivo leva a alterações no sistema cardiovascular e no aparelho locomotor. As adaptações ósseas e musculares à sobrecarga esportiva foram evidenciadas no estudo de Chang et al. (2009) pelo aumento da densidade óssea e do volume ósseo e muscular em nove atletas olímpicos de esgrima quando comparados a indivíduos fisicamente ativos. Além disso, adaptações ósseas podem ocorrer em esportes assimétricos como citado por Pereira (200 I ), que relata aumento das dimensões ósseas e da perimetria do membro superior dominante de atletas infanto-juvenis de tênis. Contudo, no presente estudo, não foi possível afirmar que o maior comprimento de membro inferior esquerdo tenha sido uma resposta adaptativa à sobrecarga mecânica do taekwondo, já que esta variável não foi monitorada longitudinalmente e os atletas avaliados não praticavam taekwondo desde a infância ou puberdade, período de maior resposta do crescimento ósseo às sobrecargas mecânicas do esporte (PEREIRA, 200 I). Também se observou que a assimetria de apenas três atletas foi superior a 1,5 cm, considerados por Magee (2002) como sendo normal. Em relação à adaptação muscular, a sobrecarga mecânica desse esporte não interferiu na perimetria de coxa, pois não houve diferença entre os hemicorpos.

Os desequilíbrios pélvicos e de membros inferiores encontrados na avaliação postural, assim como as lesões descritas neste estudo, podem ser justificadas pelas características do gesto esportivo, como a frequente utilização de rotações sobre a perna de apoio e as transferências de peso para a realização dos chutes.

Outrossim, o aperfeiçoamento de técnicas de esquiva e de ataque buscando a aplicação de menores valores de força com maior eficiência, a utilização de equipamentos de proteção, o fortalecimento muscular - buscando o equilíbrio articular adequado - e o treinamento de propriocepção podem contribuir para o aumento da performance, bem como prevenir a ocorrência de lesões e desvios posturais em atletas de taekwondo. 


\section{CONCLUSÃO}

Com base nos resultados deste estudo, conclui-se que as lesões agudas foram mais frequentes que as crônicas, incidindo principalmente nos membros inferiores, sendo a região do pé a mais acometida por entorse e fratura. Os mecanismos causadores das lesões foram o ataque (chute) e a defesa (esquiva). Foram identificadas diferenças significativas no ângulo $Q$, ângulo da perna/retropé, alinhamento horizontal da pelve e comprimento de membros entre os hemicorpos, apesar dos perímetros de coxa dos atletas não se diferenciarem.

Como forma de prevenção de lesões e desequilíbrios posturais sugere-se a inclusão sistemática de treinamento técnico preventivo, incluindo o aprimoramento dos gestos de ataque e esquiva, de exercícios de fortalecimento equilibrado dos músculos dos membros inferiores, incluindo inversores e eversores de tornozelo, exercícios compensatórios de mobilidade e flexibilidade e exercícios de propriocepção de membros inferiores.

Incidence of injuries and postural deviations in taekwondo athletes

ABSTRACT: Ten male athletes of Santa Catarina taekwondo team were evaluated with the purpose of assessing incidence of injuries and postural deviations. A questionnaire was used to access history of injuries related to the practice of this sport. Posture was evaluated by SAPO protocol. The results indicated more frequent injuries in lower limbs $(7 / 10)$, specially on feet, by fractures and sprains. Significant difference at $Q$ angle $(p=0,037)$, leg-hindfoot angle $(p=0,00 \mathrm{I})$, pelvis horizontal alignment $(p=0,016)$, and limbs length $(p=0,02)$ were identified. The relationship between postural deviations and incidence of injuries has not been established, due to the small sample size. We suggest prevention work programs and compensatory exercise to prevent injuries in taekwondo athletes.

KEYWORDS: Martial arts; injuries; posture; athletes.

Incidencia de lesiones y alteraciones posturales en atletas de taekwondo

RESUMEN: Con el objetivo de analizar la incidencia de lesiones y alteraciones posturales en el taekwondo fueron evaluados 10 atletas del sexo masculino de la seleción catarinense. El histórico de lesiones relacionadas a la práctica de esta modalidad fue obtenido por aplicación de cuestionario. La postura fue analizada por medio del protocolo del software SAPO. Los resultados indicaron lesiones más frecuentes en los miembros inferiores (7//0) principalmente en el pie, por fractura y esguince. Ocurrieron diferencias significativas entre los hemicorpos en el ángulo $Q(p=0,037)$, ángulo pierna/retropie $(p=0,00$ I $)$, alineamiento horizontal de la pelves $(p=0,016)$ y tamaño entre miembros $(p=0,02)$. Se sugiere la implantación de entrenamiento preventivo y ejercicios compensatorios para prevenir lesiones y alteraciones posturales en atletas de taekwondo.

PALABRAS CLAVE: Artes maciales; lesiones; postura; atletas. 


\section{REFERÊNCIAS}

BASTOS, F. N. et al. Correlação entre padrão postural em jovens praticantes do atletismo. Revista Brasileira de Medicina Esporte, São Paulo, v. I 5, n. 6, p. 432-435, nov./dez. 2009.

BEIS, K.; PIETER, W.; ABATZIDES, G. Taekwondo techniques and competition characteristics involved in time-loss injuries. Journal of Sports Science and Medicine, Bursa, v. 6, p. 45-5I, out. 2007.

BELCHIOR, A. C. G. et al. Efeitos na medida do ângulo $Q$ com a contração isométrica voluntária máxima do músculo quadricipital. Revista Brasileira de Medicina do Esporte, São Paulo, v. 12, n. I, p. 6-10, jan./fev. 2006.

BOUHLEL, E. et al. Heart rate and blood lactate responses during taekwondo training and competition. Science \& Sports, Paris, v. 2 I , n. 285-290, out. 2006.

BRIDGE, C. A. et al. Heart rate responses to taekwondo training in experienced practitioners. Journal of Strength and Conditioning Research, Philadelphia, v. 21, n. 3, p. 7I8-723, ago. 2007.

BRIDGE, C. A.; JONES, M. A.; DRUST, B. Physiological Responses and Perceived Exertion During International Taekwondo Competition. International Journal of Sports Physiology and Performance, Illinois, v. 4, n. 4, p. 485-493, dez. 2009.

BURKE, D. T. et al. Effect of implementation of safety measures in taekwondo competition. British Journal of sports Medicine, London, v. 37, n. 5, p. 40I-404, out. 2003.

BUSCHBACHER, R. M.; SHAY, T. Martial arts. Physical Medicine and Rehabilitation Clinics of North America, Philadelphia, v. 10, n. I, p. 35-47. 1999.

CHANG, G.; REGATTE, R. R.; SCHWEITZER, M. E. Olympic fencers: adaptations in cortical and trabecular bone determined by quantitative computed tomography. Osteoporosis International, London, v. 20, p. 779-785, may 2009.

EJNISMAN, B. et al. Lesões músculo-esqueléticas no ombro do atleta: mecanismo de lesão, diagnóstico e retorno à prática esportiva. Revista Brasileira de Ortopedia, São Paulo, v. 36, n. 10, p. 389-393, out. 2001.

ESTEVES, A. C.; REIS, D. C.; SANTOS, S. G. Impact and time of impact in bandal tchagui kick of the taekwondo. The FIEP Bulletin, Foz do Iguaçu, v. 76, p. 448-450. 2006.

FALCO, C. et al. Influence of the distance in a roundhouse kick's execution time and impact force in Taekwondo. Journal of Biomechanics, Eindhoven, v. 42, n. 3, p. 242-248, jan. 2009.

FUNDAÇÃO DE AMPARO À PESQUISA DO ESTADO DE SÃO PAULO. Software de avaliação postural - SAPO 0.68. 2003. Disponível em: <http://sapo.incubadora.fapesp.br/ portal>. Acesso em: jul. 2007. 
GARTLAND, S.; MALIK, M. H. A.; LOVELL, M. E. Injury and injury rates in Muay Thai kick boxing. Revista Brasileira de Medicina Esporte, São Paulo, v. 35, n. 5, p. 308-3। 3, out. 2001.

GUIMARÃES, M. M. B.; SACCO, I. C. N.; JOÃO, S. M. A. Caracterização postural da jovem praticante de ginástica olímpica. Revista Brasileira de Fisioterapia, São Carlos, v. I I , n. 3, p. 213-219, mai./jun. 2007.

KAZEMI, M. et al. Nine year longitudinal retrospective study of Taekwondo injuries. Journal of the Canadian Chiropractic Association, Toronto, v. 53, n. 4, p. 272-28I, dez. 2009.

KAZEMI, M.; PIETER, W. Injuries at a Canadian National Taekwondo Championships: a prospective study. BMC Musculoskeletal Disorders, London, v. 5, n. 22, jul. 2004. Disponível em: <http://www.biomedcentral.com/I47I-2474/5/22>. Acesso em: I I de jun. 2010.

KAZEMI, M.; SHEARER, H.; CHOUNG, Y. S. Pre-competition habits and injuries in Taekwondo athletes. BMC Musculoskeletal Disorders, London, v. 6, n. 26, mai. 2005. Disponível em: <http://www.biomedcentral.com/|47|-2474/6/26>. Acesso em: I I de jun. 20 I0.

LIN, W. L. et al. Anaerobic capacity of elite taiwanese taekwondo athletes. Science \& Sports, Paris, v. 2 I, p. 29I-293, jul. 2006.

LYSTAD, R. P.; POLLARD, H.; GRAHAM, P. L. Epidemiology of injuries in competition taekwondo: A meta-analysis of observational studies. Journal of Science and Medicine in Sport, Sydney, v. 12, n. 6, p. 614-621. nov. 2009.

MAGEE, D. J. Avaliação musculoesquelética. Barueri: Manole, 2002.

MELHIM, A. F. Aerobic and anaerobic power responses to the practice of taekwon-do. Revista Brasileira de Medicina do Esporte, São Paulo, v. 35, p. 23 I-234, ago. 2001.

NETO JÚNIOR, J.; PASTRE, C. M.; MONTEIRO, H. L. Alterações posturais em atletas brasileiros do sexo masculino que participaram de provas de potência muscular em competições internacionais. Revista Brasileira de Medicina do Esporte, São Paulo, v. 10, n. 3, p. 195-198, maio/jun. 2004.

OLIVEIRA, S. M.; DEPRÁ, P. P. Análise Postural: um estudo em atletas juvenis. Revista da Educação Física da UEM, Maringá, v. 16, n. 2, p. I63- I70, jul./dez. 2005.

OSBORNE, M. D.; RIZZO, T. D. J. R. Prevention and Treatment of Ankle Sprain in Athletes. Sports Medicine, Pennsylvania, v. 33, n. I5, p. I I 45- I I 50, jan. 2003.

PASTRE, C. M. et al. Sports injuries in track and field: comparison between information obtained in medical records and reported morbidity inquires. Revista Brasileira de Medicina do Esporte, São Paulo, v. 10, n. I, p. 9- I5, jan./fev. 2004.

PEREIRA, C. F. Perfil corporal de tenistas participantes do campeonato brasileiro de tênis, ambos os sexos, categoria 16 anos: um relato cineantropométrico. Revista Treinamento Desportivo, Curitiba, v. 6, n. I, p. 53-7I. 2001. 
RIBEIRO, C. Z. P. et al. Relação entre alterações posturais e lesões do aparelho locomotor em atletas de futebol de salão. Revista Brasileira de Medicina do Esporte, São Paulo, v. 9, n. 2, p. 91-97, mar./abr. 2003.

SANTOS, S. G.; PIUCCO, T.; REIS, D. C. Fatores que interferem nas lesões de atletas amadores de voleibol. Revista Brasileira de Cineantropometria e Desempenho Humano, Florianópolis, v. 9, n. I, p. 189-195, abr. 2007.

SILVA, L. R. V. Avaliação da flexibilidade e análise postural em atletas de ginástica rítmica desportiva flexibilidade e postura na ginástica rítmica. Revista Mackenzie de Educação Física e Esporte, São Paulo, v. 7, n. I, p. 59-68, abr. 2008.

WOJTYS, E. M. et al. The Association Between Athletic Training Time and the Sagittal Curvature of the Immature Spine. The American Journal of Sports Medicine, Chicago, v. 28, n. 4, p. 490-498, jul. 2000.

Recebido: 17 set. 2010 Aprovado: 05 abr. 2011

Endereço para correspondência: Aline Cavalheiro Tamborindeguy Laboratório de Biomecânica - Centro de Desportos - UFSC Campus Universitário da Trindade Florianópolis - SC CEP 88040-970 